www.jmscr.igmpublication.org

Impact Factor 5.244

Index Copernicus Value: 83.27

ISSN (e)-2347-176x ISSN (p) 2455-0450

crossref DOI:_http://dx.doi.org/10.18535/jmscr/v4i8.64

Journal Of Medical Science And Clinical Research

\title{
Detection of Diabetes In Smear Positive Pulmonary Tuberculosis Patients Attending Tertiary Care Teaching Hospital, Tamilnadu, India
}

\author{
Authors \\ T.Vijayalakshmi ${ }^{1}$, Selvaraj Stephen ${ }^{2}$, Nithyalakshmi $\mathbf{J}^{1}$, Sumathi $\mathbf{G}^{1}$, \\ Selvi $\mathbf{R}^{3}$, Jayalakshmi $\mathbf{G}^{\mathbf{4}}$. \\ ${ }^{\mathbf{1}}$ Dept of Microbiology, Sri Muthukumaran Medical college Hospital and Research Institute, \\ Chennai, Tamilnadu, India \\ ${ }^{2}$ Department of Microbiology, Mahatma Gandhi Medical college Hospital and Research institute \\ ${ }^{3}$ Department of Microbiology, Stanley Medical college Hospital and Research institute \\ ${ }^{4}$ Department of Microbiology, Annai Medical college Hospital and Research institute
}

\begin{abstract}
Background: Despite the implementation of a widely adopted strategy to control tuberculosis (TB), the disease remains a major public health problem particularly in developing country, like India. The objective of the study was to find out the prevalence of Diabetes mellitus type 11 among pulmonary tuberculosis patients in and around District of Kancheepuram, Tamil Nadu, India.

Methods: A total of 326 sputum samples were collected from clinically suspected pulmonary tuberculosis patients aged between 20-60 yrs, in a Tertiary care hospital and Research Institute Kancheepuram District, Tamilnadu, for a study period of 6 months.

Results: Among the 326 pulmonary TB suspects, a total of 53 (16.25\%) were identified as pulmonary tuberculosis based on smear positive results. All smear positive samples were subjected for Diabetes mellitus. Out of 53 TB patients, 7 (13.20\%) patients were positive for Diabetes mellitus type II. Of the 07 positive samples, 4 (57\%) males and 3 (43\%) females had Diabetes mellitus type II and PTB

Key words: Pulmonary Tuberculosis, Diabetes mellitus (DM), Sputum AFB smear, Sputum culture.
\end{abstract}

\section{INTRODUCTION}

Tuberculosis remains a major public health problem. The association between diabetes mellitus and tuberculosis and their synergistic role in causing human disease has been recognized for centuries. Tuberculosis (TB) is a major public health threat in the developing world ${ }^{(1)}$. Tuberculosis (TB) is estimated to affect $40 \%$ of the Indian population. According to $\mathrm{WHO}$, incidence of TB was reported to be 176 per 1 lakh population ${ }^{(2)}$. Each day
Tuberculosis (TB) is estimated to kill 3,500 people worldwide and one-third of the world's population is currently infected with the causative agent of TB, and 8.8 million new cases of active $\mathrm{TB}$ are estimated to occur around the world each year ${ }^{(3)}$. Diabetes Mellitus is an iceberg disease with increasing prevalence and incidence in both developed and developing countries. IDF (International Diabetes Federation) estimated that there are 382 million people worldwide living with 
Diabetes. In India, 9.1\% of the adult population (2079 years) were estimated to be living with Diabetes in 2013 and India is the largest contributor to regional (South-East Asia) mortality, with 1.1 million deaths attributable to Diabetes in $2013^{(4)}$. India is facing a double burden of both Tuberculosis and Diabetes posing a serious challenge for the health system. In recent years, strong evidence has been gathered to confirm a link between TB and yet another disease diabetes mellitus. That link had been suspected for centuries ${ }^{(5)}$. A number of studies showed higher frequencies of certain clinical findings such as lower lung field lesions, cavities and acid-fast bacilli (AFB) smear positivity among patients with TB and DM (TBDM) co morbidity ${ }^{(6-}$ 9). Adverse effects of $\mathrm{DM}$ on $\mathrm{TB}$ treatment outcomes, i.e. increased risk of death, treatment failure, default and relapse were reported in many studies $^{(10,11)}$. The objective of the study was to screen the pulmonary tuberculosis patients for type II Diabetes mellitus (DM) attending tertiary care teaching hospital, Chennai, Tamil Nadu, India.

\section{MATERIALS AND METHODS}

A total of 326 sputum samples were collected from clinically suspected pulmonary tuberculosis patients in a Tertiary care hospital and Research Institute, Chennai, Tamilnadu, India for a study period of 6 months. The study was approved by the Institutional Ethics Committee (IEC) and informed consent was obtained from each patient before enrollment for the study.

\section{Sputum collection and processing procedures}

Sputum was collected and examined for the presence of acid fast bacilli by conventional ZiehlNeelsen staining technique according to Revised National Tuberculosis Control Programme (RNTCP) diagnostic criteria ${ }^{(12)}$.

\section{Detection of type II Diabetes}

All willing participants were asked to come the next day after overnight fasting. They were subjected to fasting blood glucose (RBG) test based on a capillary blood sample with a glucometer. The fasting blood glucose levels (FBS) were measured using a standardized glucometer. Case definition for diagnosing diabetes was based on the criteria recommended by WHO ${ }^{(13) .}$ Briefly, Diabetes was identified based on diagnostic criteria of a fasting plasma glucose level of $\geq 126 \mathrm{mg} / \mathrm{dl}$.

\section{RESULTS}

Among the 326 pulmonary TB suspects, a total of $53(16.25 \%)$ were identified as smear positive for AFB. (Fig 1)

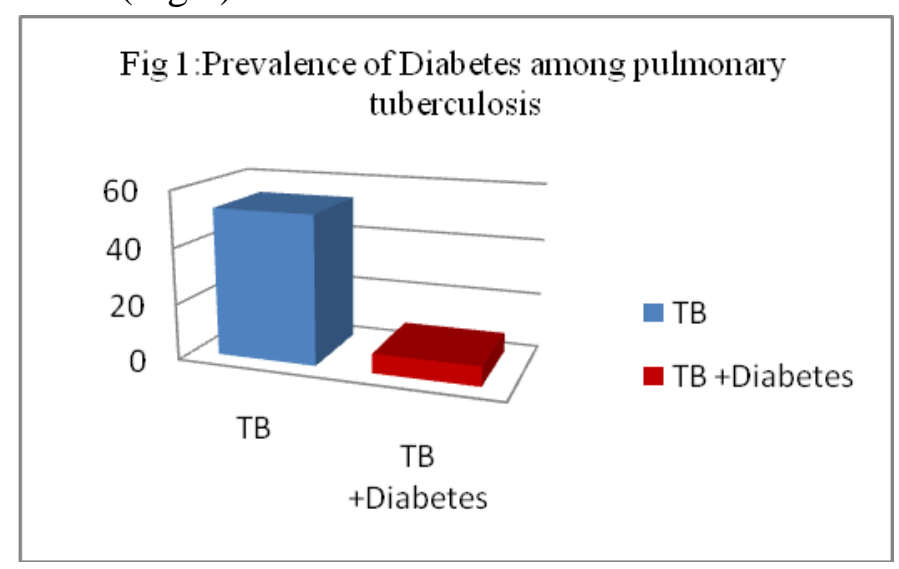

Out of 53 TB patients, 7 (13.20\%) patients had a fasting plasma glucose level of $>126 \mathrm{mg} / \mathrm{dl}$. Out the 7 tuberculosis patients with Diabetes, 4 (57\%) were males and 3 (43\%) were females (fig 2). Our study shows that PTB and Diabetes co-infection prevalence was more in males when compared to Females.

Fig 2 :Genderwise distribution of TB +Diabetes

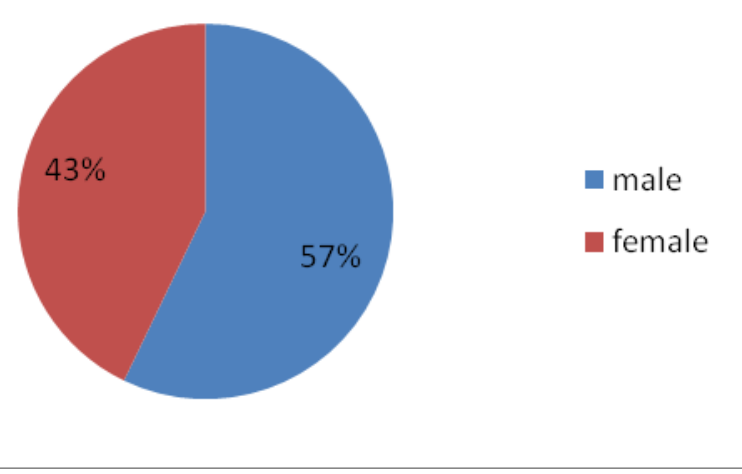

\section{DISCUSSION}

The prevalence of diabetes mellitus in India is estimated to range from 5.6 to 12.4 percent in urban area ${ }^{(14)}$. Our study shows the prevalence of $13.2 \%$ of diabetic patients among TB patients. Thus, the prevalence was found to be high among the TB patients when compared to general population. This 
was in accordance with community based cross sectional study from Tamilnadu. Recent study at Pondicherry also showed higher diabetic prevalence of $29 \%$ in TB patients $\left.{ }^{(15}\right)$. Padmalatha et al.,in a similar study at Guntur, Andhrapradesh observed $30.6 \%{ }^{(16)}$, In contrast, similar study from Gujarat had reported low prevalence ${ }^{(17)}$. This might be due to difference in prevalence percentage of $\mathrm{TB}$ with reference to geographical difference.

The prevalence was found to be more in males when compared to females. In the present study, we observed high prevalence of patients with this dual disease in the older TB patients (Age group 41$50 \mathrm{yrs}$ ). Similar findings have been observed from a
Indian study conducted by Jain et $\mathrm{al}^{(18)}$. Reports from other different countries were also in accordance with this observation. This could be attributed to the increased life expectancy due to provisions of Health services leading to increase in elderly population which would exponentially increase the cases of Diabetes.

Considering the increasing disease burden of DM, particularly in areas with highly prevalent TB, TB control programs will need to expand their efforts and focus on treating and monitoring patients with $\mathrm{DM}$ and TB disease.

Table.3 Age wise distribution of diabetes patients among smear positive pulmonary tuberculosis

\begin{tabular}{|l|c|c|c|c|}
\hline S. No & Age (in yrs) & $\begin{array}{c}\text { Smear positive } \\
\text { cases } \\
(\mathrm{n}=53)\end{array}$ & $\begin{array}{c}\text { New cases of } \\
\text { Diabetes } \\
(\mathrm{n}=7)\end{array}$ & $\begin{array}{c}\text { \% incidence of } \\
\text { diabetes among } \\
\text { TB }\end{array}$ \\
\hline 1 & $20-30$ & 14 & 1 & 7.14 \\
\hline 2 & $31-40$ & 18 & 2 & 11.11 \\
\hline 3 & $41-50$ & 21 & 4 & 19.04 \\
\hline 4 & $>51$ & 0 & 0 & 0 \\
\hline
\end{tabular}

LIMITATIONS: The limitation of the study was it included smear positive TB patients only and it is a single centered study.

\section{CONCLUSION}

This study shows that $13.2 \%$ of pulmonary TB patients had Diabetes. As diabetes can adversely affect the management of tuberculosis, we emphasize the need for timely detection of diabetes by screening TB patients routinely. This could probably improve the treatment outcome of TB patients

\section{DECLARATIONS}

Funding: None

Conflict of interest: None

Ethical approval: Obtained from institutional Ethics committee

\section{REFERENCES}

1. Niazi AK, Kalra S. Diabetes and tuberculosis: a review of the role of optimal glycemic control. J Diabetes Metab Disord. 2012;11:28.

2. World Health Organization (WHO). Global Tuberculosis report 2013.

3. World Health Organization (2011) Global tuberculosis control 2011. Geneva: WH

4. Root HF (1934) The association of diabetes and tuberculosis. N Eng J Med 210: 1-13.

5. International Diabetes Federation. IDF Diabetes Atlas, 6th edition 2013. Available from:

6. Restrepo BI, Fisher-Hoch SP, Crespo JG, Whitney E, Perza A, Smith B.Type 2 diabetes and tuberculosis in a dynamic binational border population. Epidemiol Infect. 2007;135(3):483-91. 
7. Chang JT, Dou HY, Yen CL, Wu YH, Huang RM, Lin HJ, et al. Effect of type 2 diabetes mellitus on the clinical severity and treatment outcome in patients with pulmonary tuberculosis: a potential role in the emergence of multidrug-resistance. J Formos Med Assoc. 2011;110(6):372-81.

8. Carreira S, Costeira J, Gomes C, André JM, Diogo N. Impact of diabetes on the presenting features of tuberculosis in hospitalized patients. Rev Port Pneumol. 2012;18(5):239-43.

9. Shital P, Anil J, Sanjay M, Mukund P. Tuberculosis with diabetes mellitus:clinicalradiological overlap and delayed sputum conversion needs cautious evaluationprospective cohort study in tertiary care hospital. India J Pulm Respir Med. 2014;4:175.

10. Dooley KE, Tang T, Golub JE, Dorman SE, Cronin W. Impact of diabetes mellitus on treatment outcomes of patients with active tuberculosis. Am J Trop Med Hyg. 2009;80(4):634-9.

11. Baker MA, Harries AD, Jeon CY, Hart JE, Kapur A, Lönnroth K, et al. The impact of diabetes on tuberculosis treatment outcomes: a systematic review. BMC Med. 2011;9:81.

12. New Delhi, India: Directorate General of Health Services, Ministry of Health and Family Welfare; 1998. [Last accessed on 2015 Jan 22nd]. RNTCP Central TB Division. Manual for Laboratory.

13. World Health Organization. Definition and diagnosis of diabetes mellitus and intermediate hyperglycaemia. Summary of Technical Report and Recommendations. Report of a WHO/IDF consultation. Geneva, Switzerland: WHO; 2006.

14. Ramachandran A. Epidemiology of diabetes in India: three decades of research. J Assoc Physicians India. 2005;53:34-8.

15. Manoj D. Kottarath, Rajani Mavila, et al. Prevalence of diabetes mellitus in tuberculosis patients: a hospital based study,
International Journal of Research in Medical Sciences Kottarath MD et al. Int J Res Med Sci. 2015 Oct;3(10):2810-2814

16. Dr.P.Padmalatha, Dr.K.Hema, Study on prevalence of Diabetes Mellitus in Tuberculosis patients attending a tertiary care hospital in Guntur, Andhra Pradesh, Indian Journal of Basic and Applied Medicl Research; December 2014: Vol.-4, Issue- 1, P. 494-498

17. P.Dave, A. Shah, et al. Screening patients with tuberculosis for diabetes mellitus in Gujarat, India, Public Health Action. 2013 Nov 4; 3(Suppl 1): S29-S33. 